\title{
MATLAB-Based SIMULATOR, Generating Telemetry Data Streams for Research Purposes
}

\author{
Mohamed A. Elshafey*
}

\begin{abstract}
This paper presents design and implementation of a MATLAB-based SIMULATOR for generating different telemetry data streams, based on different telemetry frame structures, in IRIG-106 telemetry standard. Presented SIMULATOR can be considered a tool for research and design of different efficient algorithms for telemetry data processing, including data compression codes, error correcting based algorithms, and frame synchronization methods. Description of different simulated telemetry signals is provided, including test patterns, harmonic signals, arbitrary digital sequences, recorded data, and filtered signals. The presented SIMULATOR supports different telemetry frame structures till commutation based on three stages of circuit switching with different frame synchronization codes in IRIG-106 standard format. The presented SIMULATOR is able to simulate the effect of imposing the telemetry data stream to Additive White Gaussian Noise (AWGN) on communication channel during transmission, as well as the effect of the noise from the on-board sensors during acquisition phase.
\end{abstract}

Keywords: Telemetry simulator, telemetry data, telemetry frame structure, IRIG-106.

\section{Introduction}

Nowadays, telemetry systems are widely used for both control and gathering information at some remote locations and transmitting data to a convenient distant location to be displayed, analyzed and recorded. The transmission media may be air and space for satellite and airborne applications, or copper wire and fiber cable for stationary ground environment $[1,2]$.

The generic telemetry system is shown in figure, Fig. 1. In data acquisition subsystem, sensors measure the amount of a physical attribute and transform the measurement to an engineering unit value. The commutator measures each of the analog voltages and outputs a single stream of pulses, each with a voltage relative to the respective measured channel (Time Division Multiplexing TDM). The analog multiplexed data is then transformed to a digital form, then modulated and transmitted via radio transmitter and antenna, coax cable, telephone line, tape recorder, etc. to a receiving station. At the receiving station, the received data stream is amplified and the de-commutator recognizes the synchronization pattern and returns the serial digital stream to parallel data $[1,2]$. 
Telemetry stream is a sequentially transmitted telemetry frames. A complete scan by the commutator (one revolution) produces data words of the telemetry frame, which contains the value of each measured parameter. Every scan produces the same sequence of words. Only a value of measured parameter is captured. For the process of de-commutation, a unique frame synchronization code is added to each frame to serve as a reference and again values of the parameters are extracted again it into individual measured values, etc. The length of the frame synchronization code is longer than usual data words to reduce the probability of actual data matching $[1,2]$.

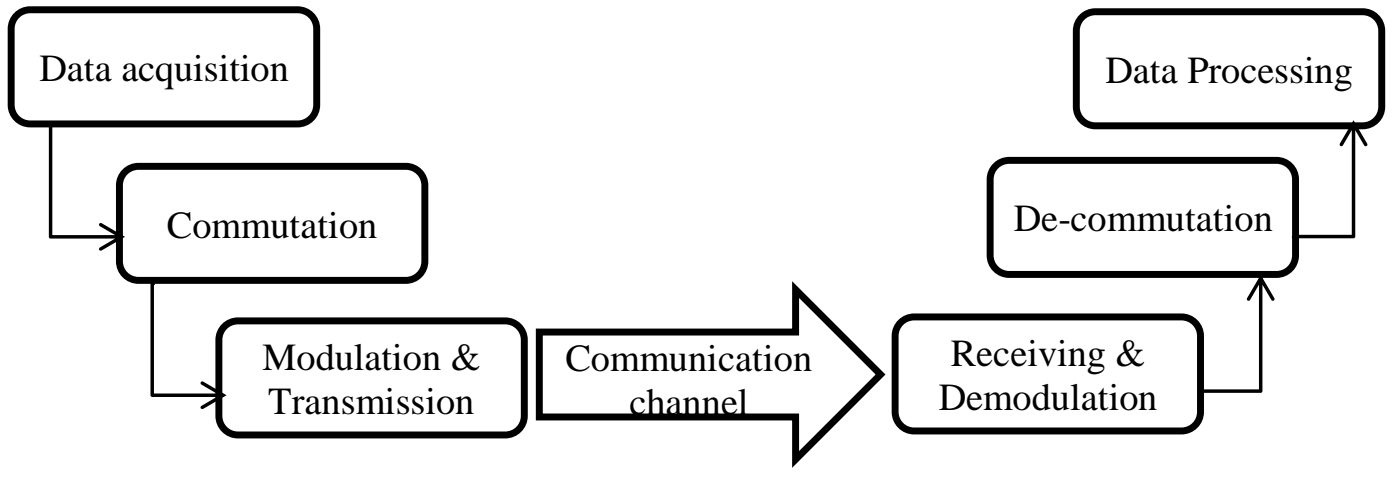

Fig. 1. Generic telemetry system

This article describes the theoretical basis and practical details of a software simulation for generation telemetry data streams for research purposes. The SIMULATOR is an important component of telemetry data registering system. As a rule, the simulators used to test individual system components at the design stage, and to validate the operation of the system before experiments at telemetry data receiving stage. The SIMULATOR also allows to simulate situations that are impossible or very expensive to reproduce in real world.

It should be noted that the functional hardware and software implementations of simulators are primarily directed to solve problems arising in the manufacture and operation of telemetric systems for various applications. In these devices, there is no functionality required for development and research purposes. Some commercially available means of registering telemetry data including embedded hardware [3,4] or software [5,6,7] simulators. These simulators allow to generate simple test data series, for example, by increasing or decreasing values of counters in all channels of the main telemetry frame. Such simulators, primarily designed to test the readiness of the system before starting reception of telemetry data. They may also be used for troubleshooting and debugging registration system during its operation.

The proposed SIMULATOR in this work is intended for research purposes for improving algorithms of real time telemetry data processing such that: telemetry data compression for transmission or achieving needs [8], implementing of error correcting codes for telemetry data during transmission over a noisy channel and also studying synchronization methods of telemetry frames in the received telemetry stream at the registering telemetry system [9]. The presented SIMULATOR is able to generate various types of signals for transmission over channels of telemetry data collection system. It can be a simple test signals, such as a constant value or a digital counter that iterates over all possible values of the commutator, or signals that simulate the telemetric information, including harmonic, non-harmonic signals as well as signals generated by linear filters. The presented SIMULATOR allows to simulate the effect of imposing to Additive White Gaussian Noise (AWGN) in transmission channel, as well as the distortion of individual parameters measured at collection stage at the on-board circuit switching and collecting of telemetry data and that is especially valuable in tests of the received 
or de-commutated data blocks, also it provides a simulation capability of failures in service information, which provides frame synchronization in telemetry stream.

The most challenging modern telemetry systems are used in aerospace research. To achieve a certain level of standardization, telemetry data streams, generated by the proposed SIMULATOR, are in accordance with standard IRIG-106 (Class I) [10,11]. It is a widely used standard in the industry, developed for representing telemetry information. It is an open standard, that is supported by Telemetry Group Organization, RCC. The standard was originally developed for use in the aerospace industry, however, now it is used in telemetry systems for various purposes. Usefulness of the standard proved its wide application in industry.

\section{Telemetry Stream in IRIG-106 Standard Format}

The standard is defined by a switching circuit of up to two stages of commutation. The frame structure is convenient in order to be able to create efficient algorithms of telemetry data processing. At the same time, it satisfies the requirements of a wide range of applications. Figure, Fig. 2. shows a circuit switching, consisting of two stages of commutation.

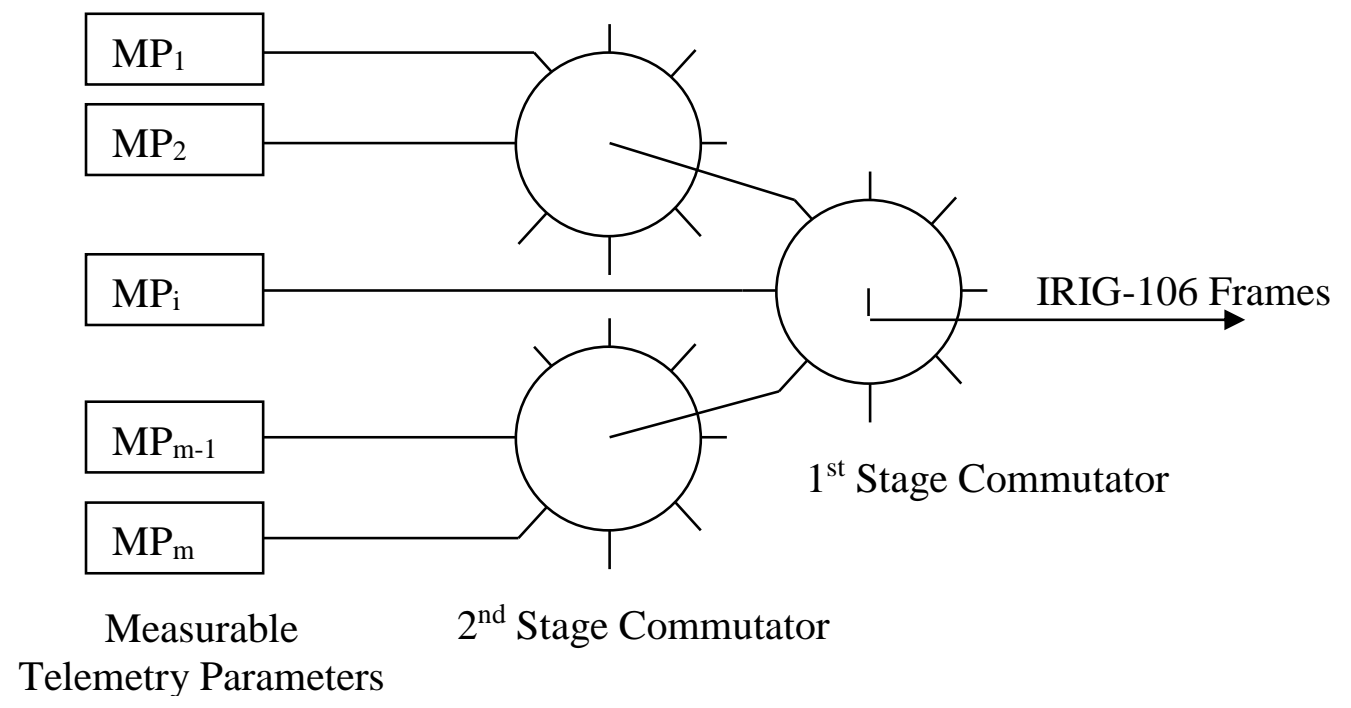

Fig. 2. Commutation scheme of telemetry information channels

All commutators in the scheme have a fixed number of channels. Single stage commutators have the same number of channels and some channels may not be used. Commutators of $1^{\text {st }}$ and $2^{\text {nd }}$ stage, tend to have a different number of channels. To this scheme, it is easy to add a $3^{\text {rd }}$ stage of commutation, in which outputs of $3^{\text {rd }}$ stage of commutation should be connected to a dedicated channel of the $2^{\text {nd }}$ stage.

The stream of telemetry data is divided into frames. Each frame contains a complete acquisition cycle of all measured parameters of the TMI system. In standard IRIG-106, that frame is called a Major_Frame. Format of the Major_Frame $[10,11]$ is shown in figure, Fig. 2, which is limited by a structure consisting of two commutation stages. 
In this figure, Fig. 3. Minor_Frame is an output frame from commutator of the $1^{\text {st }}$ stage, starting with the synchronization code (marker). Major_Frame consists of Z Minor_Frame(s). Z - is the length of the frame from commutator of the $2^{\text {nd }}$ stage. IRIG-106 standard allows $Z$ value to be not more than 256. Frame from $2^{\text {nd }}$ stage commutator is called Sub_Frame and is passed in one of the data words of the frame from $1^{\text {st }}$ stage commutator. In figure, Fig. 3. the Sub_Frame is added to data word $\mathrm{W}$.

In figure, Fig. 3. telemetry frame is composed of series of data words; each data word holds a quantized value of a sampled readout of a measurable telemetric parameter. All data words have the same bit width. For the process of de-commutation, in addition to the data words, the telemetry frame contains unique synchronization codes, which provide frame synchronization, indicating start or end of each telemetry frame for each commutator. The length of the frame's synchronization code is longer than (or at least equal to) usual data words to reduce the probability of actual data matching $[1,10]$.

The software implementation of the presented SIMULATOR is performed using MATLAB developing tool. The basis of selecting MATLAB is its ability for data processing and visualization, as well as the ability to interact with the functions and subroutines written in $\mathrm{C}$ language.

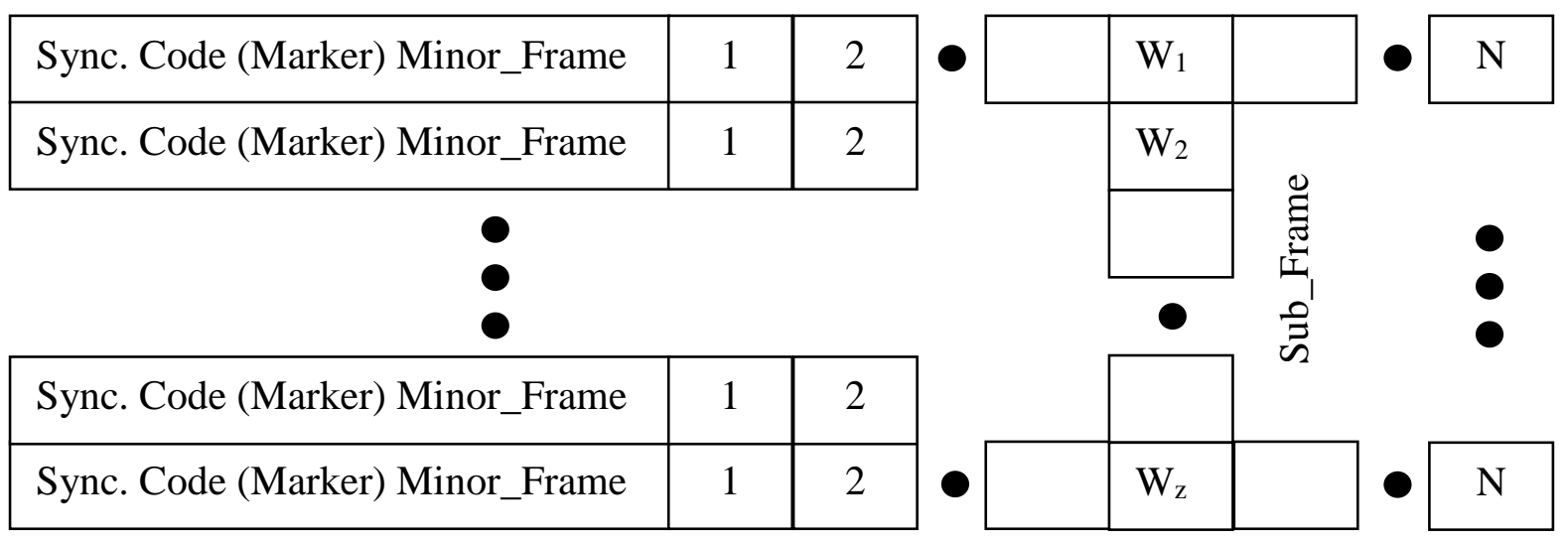

Fig. 3. Format of IRIG-106 telemetry Major_Frame

\section{Functional Parts of the Presented SIMULATOR}

The SIMULATOR consists of several software components. Each component is implemented as a separate module in MATLAB. The simulation process is divided into several steps, in each of them, single or multiple modules are involved. Each simulation project is described by a DIRECTORY of files containing all files of data and necessary describing information.

In the first step, a set of signals is generated for accommodation in channels of the commutating system. Each signal is represented on equally spaced time series, containing the digital test values or quantized values of signals, which simulate sensors' measured data. For convenience, the SIMULATOR allows to combine all data of an experiment in a single DIRECTORY. The presented DIRECTORY has many graphical user interface (GUI) forms, allowing to manage files relating to the experiment in the same project. At the end of the first stage, samples of test signals are created and stored in EXCEL files. Then, if there is no need to change the contents of these files, work on the second step can be started. 
In the second step, a desired telemetry frame structure in IRIG-106 format is created, in which telemetry test samples, from the first stage, are contained. For constructing the required telemetry frame structure, a specific GUI form is used, which allows to create up to three stages of commutating structure, of which the first two commutation stages are provided as in standard IRIG-106 and the third is implemented as described above. In the project, at this simulation step, a TEXT file is added, which contains the commutation structure of the telemetry frame format. This structure is later required to reconstruct the sequence of signals from the telemetry data frames. Additionally, in the project a BINARY file containing a binary data stream of telemetry frames, based on the previously implemented frame structure, is created. At this step of simulation, AWGN, in communication channel, can be implemented on the generated binary telemetry data stream.

At last step, the third, the original telemetry test signals can be reconstructed from the binary data stream. At this step, additional files, in EXCEL format, are created. In these files the original measured signals, transmitted over a communication channel imposed to AWGN are contained. These received samples of measured signals can be used as test data for control operations and troubleshooting software and hardware telemetry data reception, also can be implemented as inputs for telemetry data processing algorithms in different research purposes. At the end of the third step, a BINARY file containing a noised copy of the telemetry data stream is added to the project.

A brief description of the main functions the SIMULATOR is presented below.

\subsection{Generation of Telemetry Test Signals}

The signal generation module allows creation of different types of series digital values or quantized signal samples. Each such series simulates a series of data from one channel of the telemetry. This module describes the type of signal among different allowable types and additional associated parameters and settings, which specify number of quantization levels, the sampling frequency, time duration of the signal, etc. An example of such configuration for a sinusoidal signal shown in figure, Fig. 4.

The following types of signals are allowed to be generated by the SIMULATOR:

- Sine wave

$y(t)=A \sin (\omega t+\varphi)$,

- Sawtooth and triangular test signals

$y(t)=\frac{T}{2}-\frac{T}{\pi} \sum_{k=1}^{\infty} \frac{\sin (k \omega t)}{k}$,

- Rectangular pulses

$y(t)=\frac{\tau}{T}-\sum_{k=1}^{\infty} \frac{2}{k \pi} \sin \left(\frac{\pi k \tau}{T}\right) \cos \left(\frac{\pi k \tau}{T} t\right)$,

- Reaction of linear control system to a step effect.

$y(t)=\int_{-\infty}^{+\infty} h(\tau) H(t-\tau) d \tau$

- The impulse response of a linear control system.

The last two signals are primarily intended for use in engineering and scientific experiments. These types of signals are also designed for modeling, complex and difficult reproducible, in practice, scenarios' behavior of the controlled object.

Examples of telemetry test signals generated by the presented SIMULATOR are shown below. 


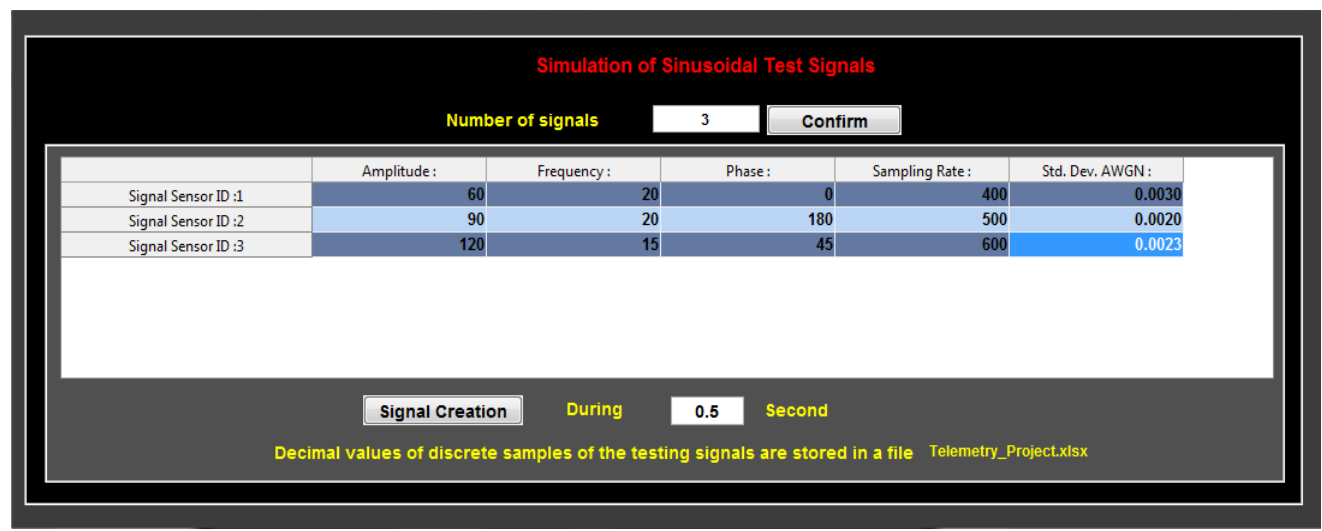

(a)

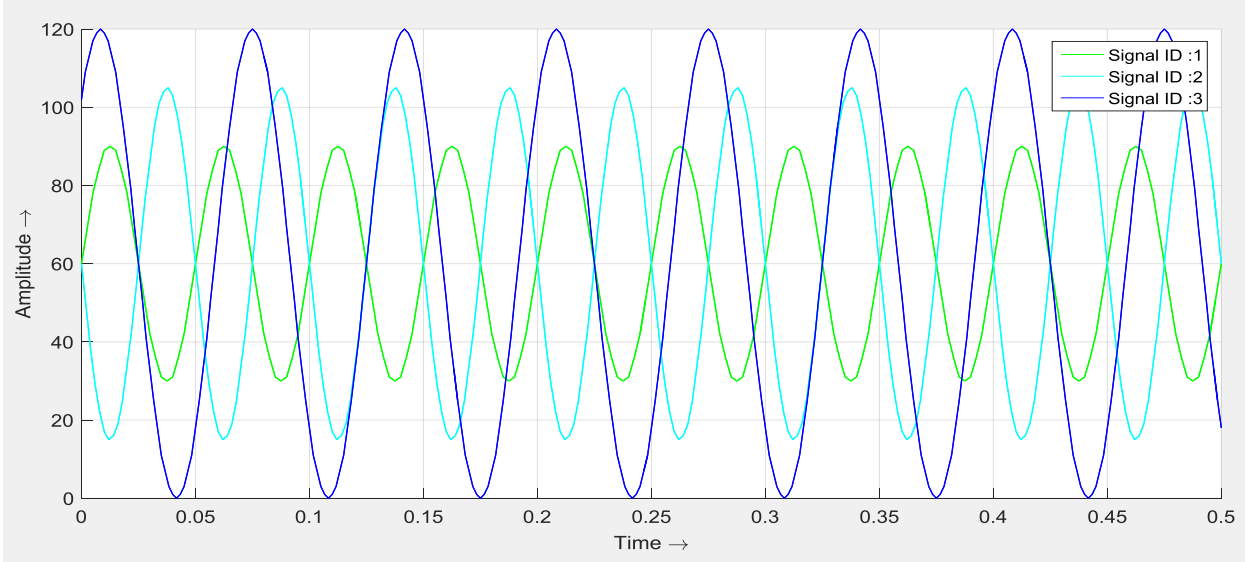

(b)

Fig. 4. Setting for (3) sinusoidal signals (a), the generated signals by the SIMULATOR (b)

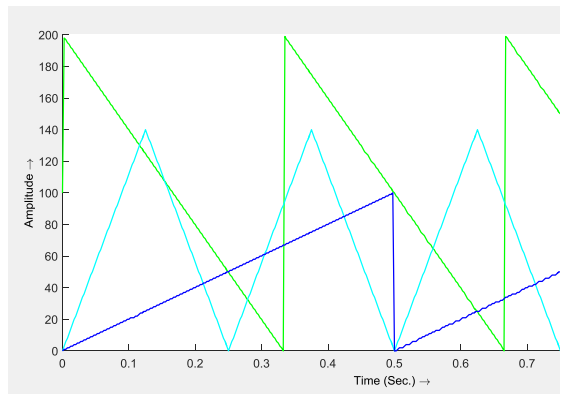

(a)

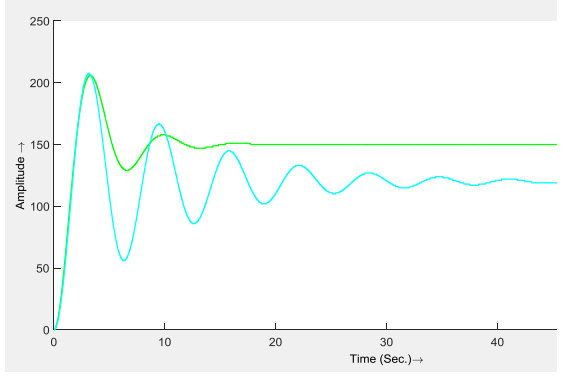

(c)

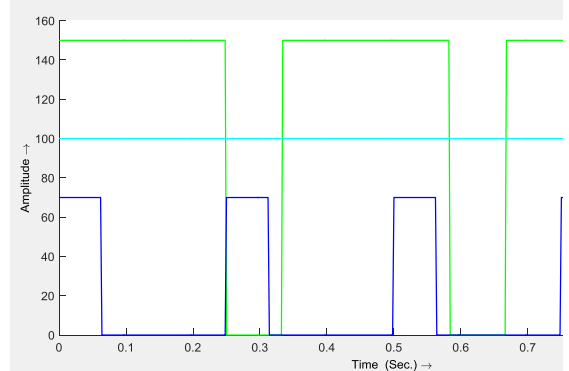

(b)

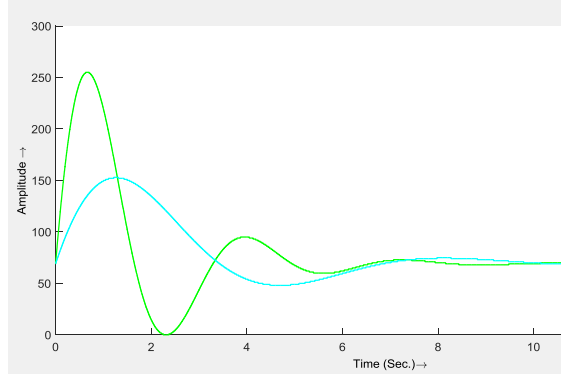

(d)

Fig. 5. Examples of generated telemetry test signals of different types: sawtooth signal and triangular shape (a), the rectangular pulse and continuous signals (b), reaction of linear control system to a step effect (c), impulse response of linear control system (d). 


\subsection{Applying Required Telemetry Frame Structure and Generating the Corresponding Binary Telemetry Data Stream}

In the proposed SIMULATOR a method for determining telemetry frame structure using a new GUI form is implemented. Description of the structure created by the operator is used to generate a binary telemetry data stream in IRIG-106 format from samples of test signals, which are generated from the previous simulation's step. The generated stream is then stored in a specific BINARY file. The frame structure is also stored in specific TEXT file. This structure is then used for the de-commutation process for the telemetry signals from the generated binary stream. Figure, Fig. 6. shows a simulation of generating a telemetry frame, which is composed of (11) channels. The Minor_Frame is composed of (8) words. The Sub_Frame (which is the generated from the $2^{\text {nd }}$ stage of commutation, which contains (3) signals from Sensor 5 , Sensor6 and Sensor7) is transmitted in the $5^{\text {th }}$ data word of the Minor_Frame. Sub_Sub_Frame (a frame generated from the commutation of the $3^{\text {rd }}$ stage, which contains (2) telemetry signals from Sensor8 and Sensor9) is passed in the $2^{\text {nd }}$ and $6^{\text {th }}$ data word of the Sub_Frame.

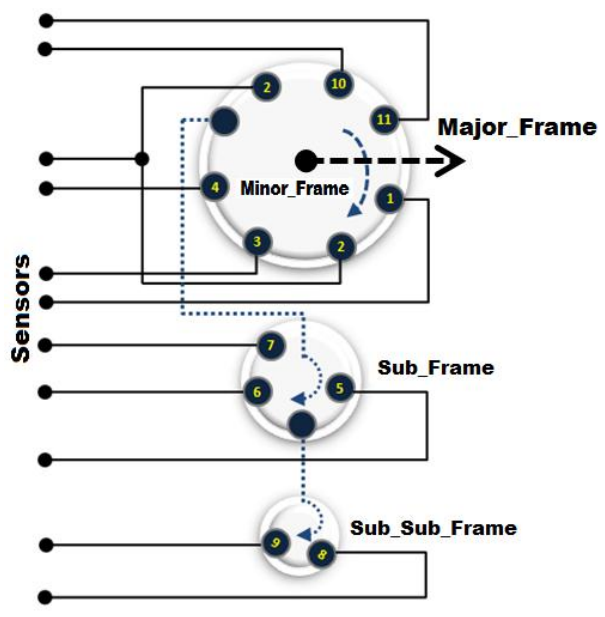

(a)

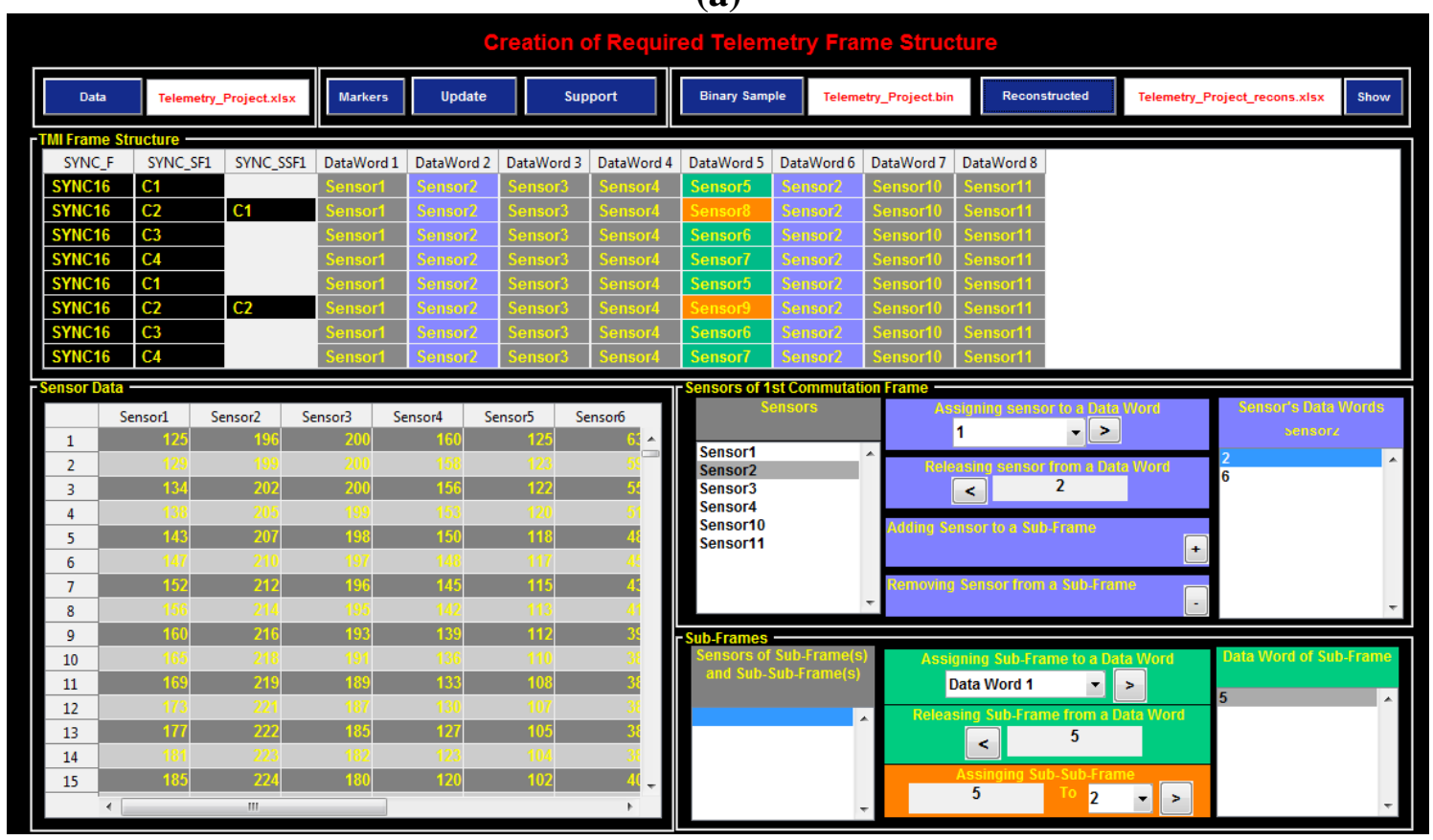

(b)

Fig. 6. Example of a commutation structure of an IRIG-106 frame: Required (a), Applied by the SIMULATOR (b) 
The SIMULATOR allows the creation of up to three stages of commutation in the implemented telemetry frame structure. Initially, in the commutation of the $1^{\text {st }}$ stage, all channels are allocated for all the test signals. All test signals are displayed in a common list and added to the Major_Frame, which is then consisting of one Minor_Frame at this step, in which all Sub_Frames are missing. Next, a list of selected test signals can be connected to the commutation of the $2^{\text {nd }}$ and $3^{\text {rd }}$ stage of commutation.

The process of implementing the structure of the telemetry frame contains elements of automation. Limitations are checked in accordance with the requirements of the standard IRIG106. Number of bits in the frame of the main commutator (Minor_Frame) and the length of the Sub_Frames are verified. A pre-selected marker (synchronization code) form a list of available (18) markers, listed in IRIG-106 standard, is automatically added to the frame of the main commutator. In Sub_Frames and Sub_Sub_Frames, counters are inserted for frame synchronization process. Standard synchronization codes for telemetry frame in IRIG-106 standard frame format are presented below.

Table 1. Markers used in IRIG-106 standard

\begin{tabular}{|c|c|}
\hline Code length (in bits) & Code Format \\
\hline 16 & $\begin{array}{llllll}111 & 010 & 111 & 001 & 000 & 0\end{array}$ \\
\hline 17 & $\begin{array}{llllll}111 & 100 & 110 & 101 & 000 & 00\end{array}$ \\
\hline 18 & $\begin{array}{llllll}111 & 100 & 110 & 101 & 000 & 000\end{array}$ \\
\hline 19 & $\begin{array}{lllllll}111 & 110 & 011 & 001 & 010 & 000 & 0\end{array}$ \\
\hline 20 & $\begin{array}{lllllll}111 & 011 & 011 & 110 & 001 & 000 & 00\end{array}$ \\
\hline 21 & $\begin{array}{lllllll}111 & 011 & 101 & 001 & 011 & 000 & 000\end{array}$ \\
\hline 22 & $\begin{array}{llllllll}111 & 100 & 110 & 110 & 101 & 000 & 000 & 0\end{array}$ \\
\hline 23 & $\begin{array}{llllllll}111 & 101 & 011 & 100 & 110 & 100 & 000 & 00\end{array}$ \\
\hline 24 & $\begin{array}{llllllll}111 & 110 & 101 & 111 & 001 & 100 & 100 & 000 \\
\end{array}$ \\
\hline 25 & $\begin{array}{lllllllll}111 & 110 & 010 & 110 & 111 & 000 & 100 & 000 & 0\end{array}$ \\
\hline 26 & $\begin{array}{lllllllll}111 & 110 & 100 & 110 & 101 & 100 & 110 & 000 & 00\end{array}$ \\
\hline 27 & $\begin{array}{lllllllll}111 & 110 & 101 & 101 & 001 & 100 & 110 & 000 & 000\end{array}$ \\
\hline 28 & $\begin{array}{llllllllll}111 & 101 & 011 & 110 & 010 & 110 & 011 & 000 & 000 & 0\end{array}$ \\
\hline 29 & $\begin{array}{llllllllll}111 & 101 & 011 & 110 & 011 & 001 & 101 & 000 & 000 & 00\end{array}$ \\
\hline 30 & $\begin{array}{llllllllll}111 & 110 & 101 & 111 & 001 & 100 & 110 & 100 & 000 & 000 \\
\end{array}$ \\
\hline 31 & $\begin{array}{lllllllllll}111 & 111 & 100 & 110 & 111 & 110 & 101 & 000 & 010 & 000 & 0\end{array}$ \\
\hline 32 & $\begin{array}{lllllllllll}111 & 111 & 100 & 110 & 101 & 100 & 101 & 000 & 010 & 000 & 00\end{array}$ \\
\hline 33 & $\begin{array}{lllllllllll}111 & 110 & 111 & 010 & 011 & 101 & 001 & 010 & 010 & 011 & 000\end{array}$ \\
\hline
\end{tabular}

Fig. 7. shows an example of the TEXT file, which contains description of a telemetry frame structure, that is created by the presented SIMULATOR.

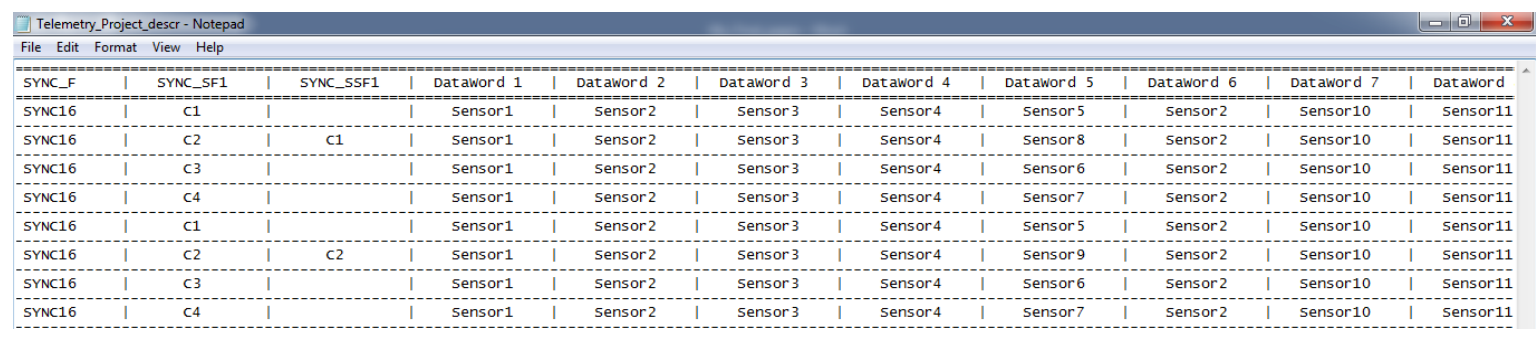

Fig. 7. Example of a TEXT file describing a telemetry frame structure 
The length of the Major_Frame (expressed in bits), of the above example, is calculated as:

$$
L_{\text {ok }}=8 L_{\text {sync_f }}+8 L_{\text {sync_sf } 1}+2 L_{\text {sync_ssf } 1}+64 L_{\text {data }},
$$

where $L_{\text {sync_xx }}$ is the number of bits used to represent synchronization codes of different commutation stages; $L_{s y n c_{-} f}-$ marker for each Minor_Frame, $L_{s y n c_{-} f f_{1}}-$ marker of the Sub_Frame, $L_{\text {sync_ssf } 1}$ - marker of the Sub_Sub_Frame and $L_{\text {data }}$ - length of a telemetry data word in bits.

\subsection{Simulation of AWGN in the Communication Channel}

The SIMULATOR uses a mathematical model of the communication channel [12], in which signal to noise ratio (SNR) of binary channel with additive Gaussian noise is defined by:

$$
S N R=\frac{E_{b}}{N_{0}}=\frac{\mu^{2}}{2 \sigma^{2}},
$$

where $E_{b}-$ bit energy, $N_{0}-$ The noise power spectral density. parameter $\mu=1$, then SNR depends only on the variance $\sigma^{2}$.

Based on the above theoretical model, algorithm is developed to simulate the effect of imposing telemetry stream to AWGN in the communication channel. Input for this process is extracted from the BINARY file, which contains the original telemetry data in standard IRIG-106 frame format. Simulation's result is also stored in an additional BINARY file.

Noise modeling procedure in the communication channel is presented below.

$$
\begin{aligned}
& \text { function Noised_Data=Add_Noise(Data,BER) } \\
& E b N O=\left(\operatorname{erfcinv}\left(B E R^{*} 2\right)\right)^{\wedge} 2 ; \quad \% \text { erfinv }()-\text { the inverse complementary error function } \\
& N O=1 / E b N O \\
& \% E b=1 \\
& \text { var }=n 0 / 2 \text {; } \\
& \text { sigma }=\text { sqrt(var); } \\
& \% \text { Variance }=\mathrm{NO} / 2 \\
& \text { Data }=2 * \text { Data }-1 ; \quad \% \text { Binary Phase Shift Keying } 0 \text {-> -1; } 1 \text {-> } 1 \\
& \text { Noise }=\text { sigma } * \text { randn(length }(\text { Data }), 1) ; \quad \% \text { Generation of White Gaussian Noise with a } \\
& \% \text { given standard deviation (sigma) } \\
& \text { Noised_Data }=\text { Data }+ \text { Noise } ; \quad \% \text { Adding AWGN to Data } \\
& \text { Noised_Data }=\text { Noised_Data }>0 \text {; }
\end{aligned}
$$

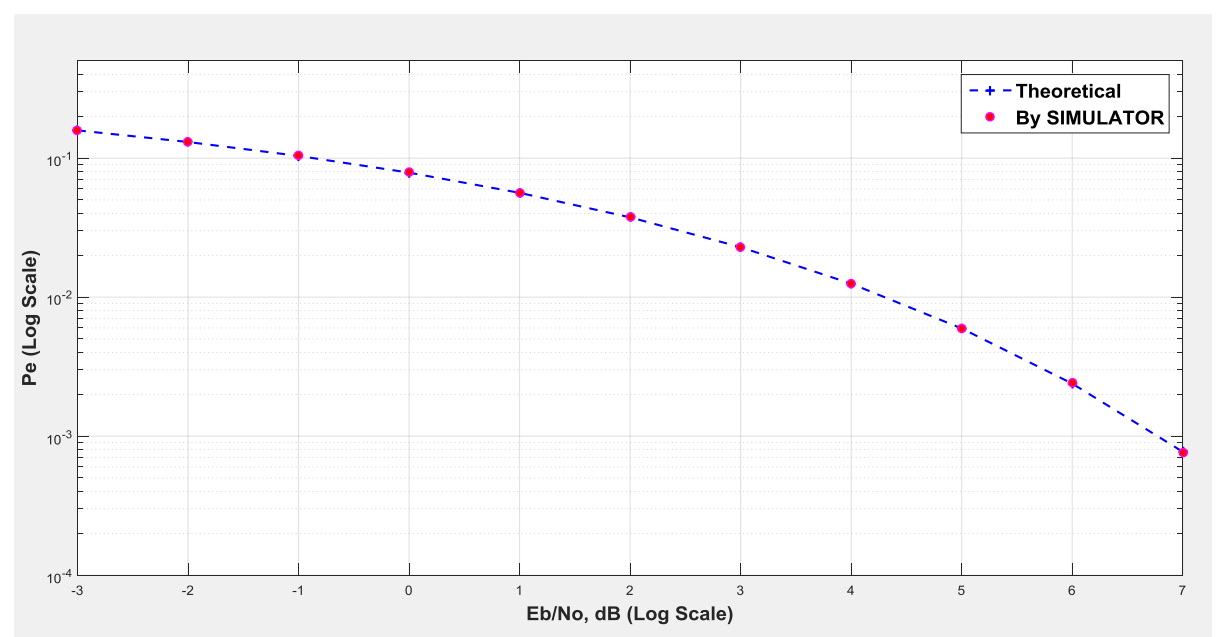

Fig. 8. BER Curve: Theoretical and by SIMULATOR

Parameter BER in the presented algorithm determines bit error rate. This value is determined by the formula:

$$
B E R=\frac{1}{2} \operatorname{erfc}\left(\sqrt{\frac{E_{b}}{N_{o}}}\right),
$$


where $\operatorname{erfc}$ - error function, which is defined by:

$$
\operatorname{erfc}=\frac{2}{\sqrt{\pi}} \int_{x}^{\infty} e^{-t^{2}} d t
$$

As a result of this algorithm, a BINARY file containing a noisy binary telemetry data stream, is added to the simulation project.

\subsection{Reconstruction of Telemetry Signals}

For the reconstruction process of the telemetry signals from telemetry data stream, the TEXT file, containing the structure of the generated telemetry frames, is used. Using information from this file, the SIMULATOR de-commutates binary data stream and extracts telemetry signals. The SIMULATOR has a GUI, which allows to analyze and compare the original telemetry signal and the de-commutated one. The reconstructed signals can be saved for further postprocessing.

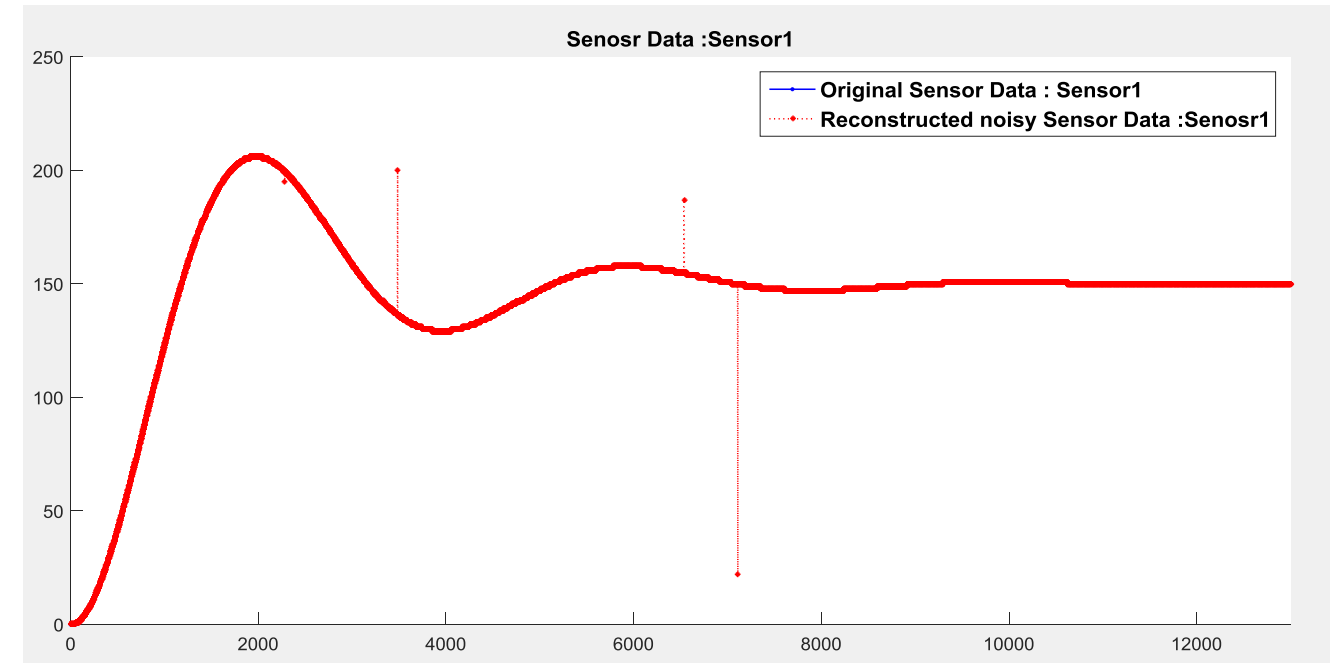

Fig. 9. Reconstructed test signal with noise effect

\section{Conclusion}

The paper presents a brief analysis of the scope of telemetry simulators. The urgency of developing a telemetry SIMULATOR expanded in the research field of designing algorithms for telemetry data processing, as well as for testing and debugging developed components and operated telemetry systems. The presented SIMULATOR allows to generate telemetry data streams of different types and even construct a required stream from previously created and/or measured signals, based on required telemetry frame structure, which makes the SIMULATOR an appropriated tool to simulate complex scenarios of object behavior, including unexpected situations, which are considered too costly, difficult and may be impossible for their reproduction for research or test purposes. The choice IRIG-106 standard format comes from it's widely usage in commercial and academic researches. In addition to the availability of commutation up to two stages, supported by IRIG-106 standard, the SIMULATOR allows up to three stages of commutation for more research purposes. Described, in paper, the simulation steps of transmitting a telemetry data stream over a noisy channel and reconstruction of the signals from the received one. 


\section{References}

[1] Carden F., Jedlicka R., Henry R, Telemetry Systems Engineering. London (UK): Artech House, 2002, 622 p.

[2] Horan S., Introduction to PCM Telemetering Systems. $2^{\text {nd }}$ Edition. USA: CRC Press, 2002, 448 p.

[3] TelSim Telemetry Simulator. CCE Corp. URL: http://cctcorp.com/products/telemetryacquisition-simulation/telsim/

[4] MAG_200/SIM_RF Mobile RF Telemetry Simulation Station Data Sheet. ADAS URL: http://www.magali.com/telemesure/pdf/MAG_200_SIM-RF.pdf.

[5] TarsusHS-PCI-01 33Mbps PCM Processor Data Sheet. ULYSSIX Technologies. URL: http://www.ulyssix.com/Downloads/Brochures/TarsusHS-PCI-01.pdf.

[6] ALSEP Telemetry Data Simulation. Aerospace System Division. 1967, pp. 43 URL: http://www.lpi.usra.edu/lunar/ALSEP/pdf/ALSEP\%20\%23222\%20\%20Telemetry\%20Data\%20Sim_ATM\%20646.pdf

[7] Douglas C. et al. Virtual telemetry for dynamic data-driven application simulations // ICCS'03 Proc. of ICCS. Berlin: Springer Verlag, 2003. pp. 279-288.

[8] Lossless Data Compression. Report Concerning Space Data System Standard, Informational Report CCSDS 120.0 G-3 Green Book. 2013.

[9] TM Synchronization and Channel Coding-Summary of Concept and Rationale. Report Concerning Space Data System Standard. Informational Report CCSDS 130.1-G-2. Green Book. Issue 2. November 2012. Washington, DC, USA, CCSDS, 2012.

[10] Telemetry Standards, IRIG Standard 106-13. New Mexico: Secretariat Range Commanders Council US Army White Sand Missile Range. 2013. URL:http://www.irig106.org/docs/106-13/

[11] IRIG 106. Chapter 10 Programming Handbook. New Mexico: Secretariat Range Commanders Council U.S. Army White Sands Missile Range. URL: http://www.irig106.org/docs/123-09/IRIG_106 07_Chapter_10_Programming_Handbook.pdf.

[12] Johnson S. J. Iterative Error Correction: Turbo, Low Density Parity Check and Repeat Accumulate Codes. Cambridge University Press. 2009. 
Flowchart of the functional parts of the presented Simulator

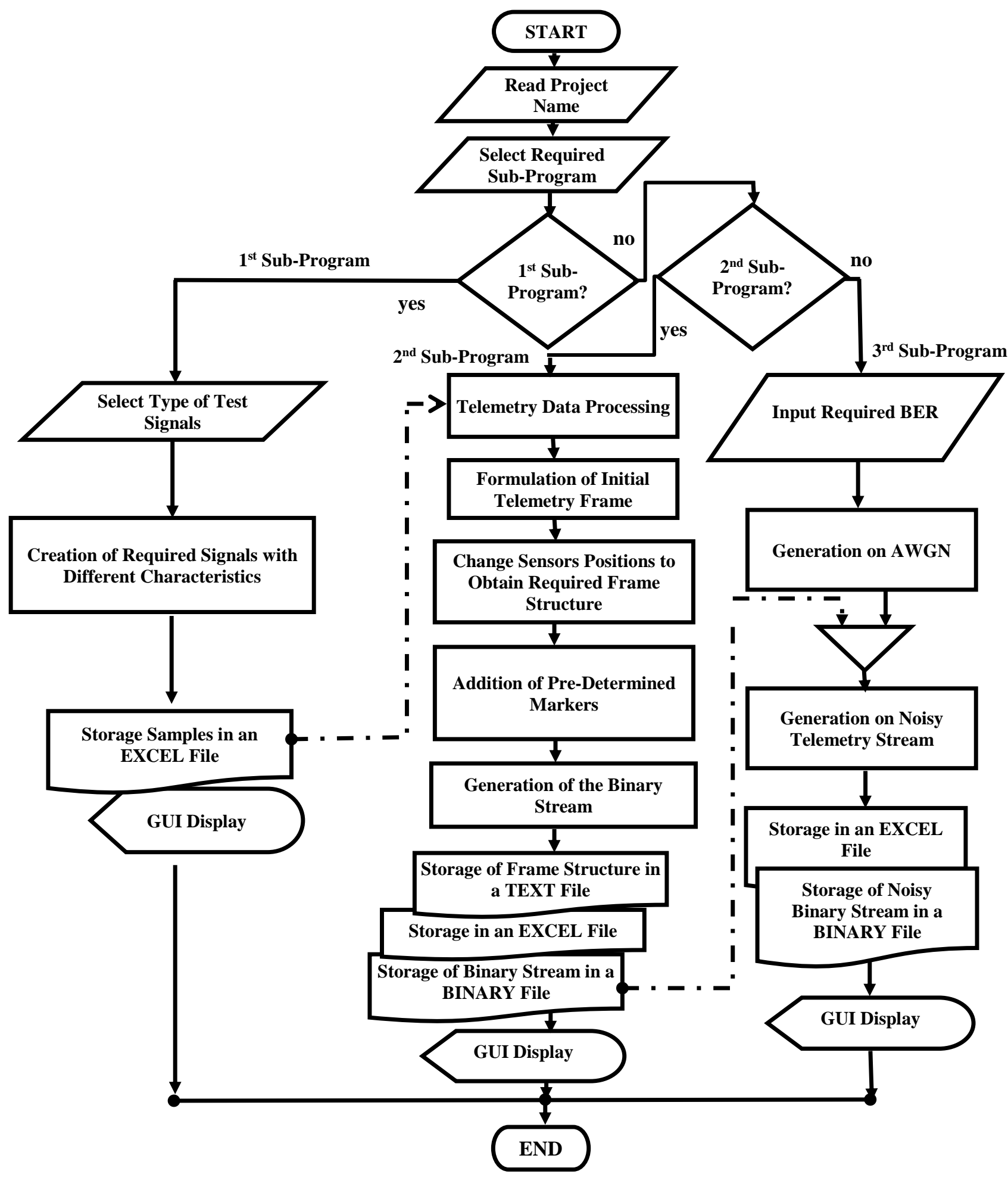

